\title{
ON PARABOLIC EQUATIONS FOR MEASURES
}

\author{
VLADIMIR I. BOGACHEV, GIUSEPPE DA PRATO, AND MICHAEL RÖCKNER
}

Abstract. A new existence result is established for weak parabolic equations for probability measures. A priori estimates for solutions of such equations are obtained.

AMS Subject Classification: 35K10, 35K12, 60J35, 60J60, 47D07

\section{INTRODUCTION}

This work is continuation of our paper [1], where we studied parabolic equations of the form

$$
L^{*} \mu=0
$$

for Borel measures $\mu$ on $(0,1) \times \mathbb{R}^{d}$. Here $L$ is a second order parabolic operator

$$
L u(t, x):=\frac{\partial u(t, x)}{\partial t}+a^{i j}(t, x) \partial_{x_{i}} \partial_{x_{j}} u(t, x)+b^{i}(t, x) \partial_{x_{i}} u(t, x),
$$

and the interpretation of our equation is the following. We shall say that a family of Radon measures $\mu=\left(\mu_{t}\right)_{t \in[0,1)}$ on $\mathbb{R}^{d}$ satisfies the weak parabolic equation (1.1) if the functions $a^{i j}$ and $b^{i}$ are integrable on every compact set in $(0,1) \times \mathbb{R}^{d}$ with respect to the measure $\mu(d t d x):=\mu_{t}(d x) d t$ and, for every $u \in C_{0}^{\infty}\left((0,1) \times \mathbb{R}^{d}\right)$, one has

$$
\int_{0}^{1} \int_{\mathbb{R}^{d}} L u(t, x) \mu_{t}(d x) d t=0
$$

We shall say that $\mu$ satisfies the initial condition $\mu_{0}:=\nu$ at $t=0$ if $\nu$ is a measure on $\mathbb{R}^{d}$ and

$$
\lim _{t \rightarrow 0} \int_{\mathbb{R}^{d}} \zeta(x) \mu_{t}(d x)=\int_{\mathbb{R}^{d}} \zeta(x) \nu(d x)
$$

for all $\zeta \in C_{0}^{\infty}\left(\mathbb{R}^{d}\right)$. In this case we write $\mu=\left(\mu_{t}\right)_{t \in[0,1)}$.

The same definitions are introduced in the case where $\mathbb{R}^{d}$ is replaced by an open set $\Omega \subset \mathbb{R}^{d}$ or by an open set in a Riemannian manifold. In particular, in (1.2) we take $u \in C_{0}^{\infty}((0,1) \times \Omega)$ and in $(1.3)$ we take $\zeta \in C_{0}^{\infty}(\Omega)$.

Equation (1.1) is satisfied for the transition probabilities of the diffusion process with the diffusion matrix $\sqrt{2 A}$ and drift $b$ provided such a diffusion exists and the coefficients $A$ and $b$ satisfy certain conditions. However, (1.1) can be considered regardless of any probabilistic assumptions. Moreover, a study of this equation in a purely analytic setting may be useful for constructing an associated diffusion (see [10], [14], [15]).

As compared to [1], the principal novelty of this work is a considerably weaker sufficient condition for the existence of solutions. Namely, in addition to certain mild local restrictions on the coefficients $A$ and $b$ it is only required that the estimate $L \Psi \leq C+C \Psi$ should hold for some compact positive function $\Psi$. In [1, Theorem 3.1], a much more restrictive additional assumption was imposed that, for each $\tau \in(0,1)$, there is a compact function $V_{\tau}$ with $L V_{\tau}(t, x) \rightarrow-\infty$ as $|x| \rightarrow \infty$. Also, our approach in this work differs considerably from that of [1], where the time-dependent 
coefficients $A$ and $b$ were approximated by piece-wise constant (in $t$ ) coefficients and the results from [2], [3] and [8] on the semigroups generated by time-independent elliptic operators were employed. Here we obtain solutions as limits of solutions to boundary value problems in bounded domains. To this end, certain a priori estimates for such solutions are obtained. In this respect, our approach is closer to that of [4], [5], [6] and [9] in the elliptic case.

\section{Auxiliary Results}

Let us recall some standard notation for various Sobolev classes on $\mathbb{R}^{d}$ or on open sets $U \subset \mathbb{R}^{d}$. The class $H^{p, 1}(U)$ consists of all functions $f \in L^{p}(U)$ with generalized partial derivatives $\partial_{x_{i}} f \in L^{p}(U)$. This space is equipped with its natural Sobolev norm $\|f\|_{p, 1}$.

For a function $u$ on $(0,1) \times \mathbb{R}^{d}$, we set $\partial_{t} u(t, x):=\partial u(t, x) / \partial t$.

We shall say that a function $V$ on $\mathbb{R}^{d}$ is compact if $\lim _{|x| \rightarrow+\infty} V(x)=+\infty$.

Lemma 2.1. If $\mu=\left(\mu_{t}\right)_{t \in[0,1)}$ satisfies (1.1) and (1.3), then, for every $\zeta \in C_{0}^{\infty}\left(\mathbb{R}^{d}\right)$, for almost all $t \in[0,1)$ one has

$$
\int_{\mathbb{R}^{d}} \zeta(x) \mu_{t}(d x)-\lim _{\varepsilon \rightarrow 0} \int_{\varepsilon}^{t} \int_{\mathbb{R}^{d}} L \zeta(s, x) \mu_{s}(d x) d s=\int_{\mathbb{R}^{d}} \zeta(x) \nu(d x) .
$$

If, for each $\zeta \in C_{0}^{\infty}\left(\mathbb{R}^{d}\right)$, the function $\int_{\mathbb{R}^{d}} \zeta(x) \mu_{t}(d x)$ is continuous on $[0,1)$, then (2.1) holds for all $t \in[0,1)$ and is equivalent to (1.1) and (1.3). The same is true in the case when our equation is considered on an open set.

This lemma was stated in [1] with the integral over $[0, t]$ in place of the limit of the integrals over $[\varepsilon, t]$, but the proof only gives the above assertion (which is sufficient for the subsequent application of the lemma in [1]). Note that the function

$$
h: s \mapsto \int_{\mathbb{R}^{d}} L \zeta(s, x) \mu_{s}(d x)
$$

is integrable on $[0, t]$ (so that the limit of the integrals over $[\varepsilon, t]$ equals the integral over $[0, t])$ provided that there is a $\mu$-integrable function $\Theta$ such that $L \zeta(s, x) \leq$ $\Theta(s, x) \mu$-a.e. Indeed, in this case the function $h$, which coincides with the derivative of the function

$$
f(s):=\int_{\mathbb{R}^{d}} \zeta(x) \mu_{s}(d x)
$$

on $(0,1)$, is majorized by the integrable function $s \mapsto \int_{\mathbb{R}^{d}} \Theta(s, x) \mu_{s}(d x)$, which yields that $f$ is absolutely continuous provided it is continuous. If we do not assume the continuity of $f$, then we conclude that $f$ has an absolutely continuous modification. Certainly, all this is true if the functions $a^{i j}$ and $b^{i}$ are $\mu$-integrable on every set $[0,1] \times B$, where $B$ is a ball in $\mathbb{R}^{d}$.

It is worth noting that one of the reasons why we require that all measures $\mu_{t}$ (and not just almost all) be probabilities is that this is the case when one deals with transition probabilities. From the analytic point of view, this is not essential, of course. Another reason is that, as we shall see, this assumption simplifies certain technical issues (see also [7]).

The following lemma is a straightforward extension of [1, Lemma 2.2] where $M=0$ and $\Theta$ is a constant. 
Lemma 2.2. Let $\mu=\left(\mu_{t}\right)_{t \in[0,1)}$ be a family of probability measures on $\mathbb{R}^{d}$ satisfying (1.1) and (1.3), where $\nu$ is a probability measure on $\mathbb{R}^{d}$. Suppose that there exist a nonnegative function $\Psi \in C^{2}\left(\mathbb{R}^{d}\right)$ and a number $M$ such that $\Psi \in L^{1}(\nu)$, $\lim _{|x| \rightarrow \infty} \Psi(x)=+\infty$, and

$$
L \Psi(t, x) \leq \Theta(t, x)+M \Psi(x) \quad \mu_{t} d t \text {-a.e. }
$$

for some $\mu$-integrable function $\Theta$. Then, for a.e. $t \in[0,1)$, one has

$$
\begin{aligned}
& \int_{\mathbb{R}^{d}} \Psi d \mu_{t} \leq \int_{\mathbb{R}^{d}} \Psi d \nu+\int_{0}^{t} \int_{\mathbb{R}^{d}} \Theta d \mu_{s} d s \\
&+M \exp (M t) \int_{0}^{t} \exp (-M s)\left[\int_{\mathbb{R}^{d}} \Psi d \nu+\int_{0}^{s} \int_{\mathbb{R}^{d}} \Theta d \mu_{r} d r\right] d s \\
& \leq\left(M e^{M}+1\right)\left[\|\Psi\|_{L^{1}(\nu)}+\|\Theta\|_{L^{1}(\mu)}\right] .
\end{aligned}
$$

If $M=0$ and $\Theta=K$ is constant, then, for a.e. $t \in[0,1)$, one has

$$
\int_{\mathbb{R}^{d}} \Psi(x) \mu_{t}(d x) \leq t K+\int_{\mathbb{R}^{d}} \Psi(x) \nu(d x) .
$$

Furthermore, if the functions

$$
t \mapsto \int \zeta(x) \mu_{t}(d x), \quad \zeta \in C_{0}^{\infty}\left(\mathbb{R}^{d}\right)
$$

are continuous on $[0,1)$, then (2.3) and, in the case $M=0,(2.4)$ are true for all $t \in[0,1)$. Finally, if (1.1) and (1.3) are fulfilled on the open set $\{\Psi<c\}$, then the same assertions with $\mathbb{R}^{d}$ replaced by $\{\Psi<c\}$ are true.

Proof. It is clear that (2.1) remains true also for any function $\zeta \in C_{b}^{\infty}\left(\mathbb{R}^{d}\right)$ such that $\zeta(x)=q=$ const outside some ball. Indeed, the function $\zeta_{0}:=\zeta(x)-q$ is of compact support, $L \zeta_{0}=L \zeta$ and

$$
\int_{\mathbb{R}^{d}} q \mu_{t}(d x)=\int_{\mathbb{R}^{d}} q \nu(d x)=q .
$$

Furthermore, due to the local integrability of the functions $a^{i j}$ and $b^{i}$ with respect to $\mu,(2.1)$ is clearly still true for $\zeta \in C_{b}^{2}\left(\mathbb{R}^{d}\right)$ (in place of $C_{0}^{\infty}$ ) such that $\zeta(x)=q=$ const outside some ball. Now let us fix $k \in \mathbb{N}$ and take a function $\theta_{k} \in C^{2}(\mathbb{R})$ such that $\theta_{k}(r)=r$ if $r \leq k, \theta_{k}(r)=k+1$ if $r \geq k+2,0 \leq \theta_{k}^{\prime}(r) \leq 1$, and $\theta_{k}^{\prime \prime}(r) \leq 0$. By our assumption on $\Psi, \theta_{k} \circ \Psi$ is constant outside a sufficiently large ball. Hence, as explained above, (2.1) is true with $\zeta(x)=\zeta_{k}(x):=\theta_{k}(\Psi(x))$. We observe that

$$
L \zeta_{k}=\theta_{k}^{\prime}(\Psi) L \Psi+\theta_{k}^{\prime \prime}(\Psi)\langle A \nabla \Psi, \nabla \Psi\rangle \leq \theta_{k}^{\prime}(\Psi) L \Psi .
$$

Hence, for a.e. $s$, one has

$$
\begin{aligned}
\int_{\mathbb{R}^{d}} L \zeta_{k}(s, x) \mu_{s}(d x) \leq \int_{\mathbb{R}^{d}}\left[|\Theta(s, x)|+M \theta_{k}^{\prime}(\Psi(x)) \Psi(x)\right] \mu_{s}(d x) & \\
& \leq(k+1) M+\int_{\mathbb{R}^{d}}|\Theta(s, x)| \mu_{s}(d x) .
\end{aligned}
$$

By our hypothesis, the right-hand side is integrable in $s$ over $[0,1]$. Note also that $\theta_{k}^{\prime}(\Psi) \Psi \leq \theta_{k}(\Psi)$. Indeed, $\left(\theta_{k}^{\prime}(t) t\right)^{\prime}=\theta_{k}^{\prime}(t)+\theta_{k}^{\prime \prime}(t) t \leq \theta_{k}^{\prime}(t)$. Therefore, for a.e. 
$t \in[0,1)$ one has

$$
\begin{aligned}
\int_{\mathbb{R}^{d}} \theta_{k} \circ \Psi d \mu_{t} & =\int_{\mathbb{R}^{d}} \theta_{k} \circ \Psi d \nu+\int_{0}^{t} \int_{\mathbb{R}^{d}} L \zeta_{k} d \mu_{s} d s \\
& \leq \int_{\mathbb{R}^{d}} \theta_{k} \circ \Psi d \nu+\int_{0}^{t} \int_{\mathbb{R}^{d}}\left(\theta_{k}^{\prime} \circ \Psi\right) L \Psi d \mu_{s} d s \\
& \leq \int_{\mathbb{R}^{d}} \Psi d \nu+\int_{0}^{t} \int_{\mathbb{R}^{d}}\left(\theta_{k}^{\prime} \circ \Psi\right)[\Theta+M \Psi] d \mu_{s} d s \\
& \leq \int_{\mathbb{R}^{d}} \Psi d \nu+\int_{0}^{t} \int_{\mathbb{R}^{d}}\left[\left(\theta_{k}^{\prime} \circ \Psi\right) \Theta+M \theta_{k} \circ \Psi\right] d \mu_{s} d s .
\end{aligned}
$$

By Gronwall's inequality we find

$\int_{0}^{t} \int_{\mathbb{R}^{d}} \theta_{k} \circ \Psi d \mu_{s} d s \leq \exp (M t) \int_{0}^{t} \exp (-M s)\left[\int_{\mathbb{R}^{d}} \Psi d \nu+\int_{0}^{s} \int_{\mathbb{R}^{d}}\left(\theta_{k}^{\prime} \circ \Psi\right) \Theta d \mu_{r} d r\right] d s$.

Hence

$$
\begin{aligned}
\int_{\mathbb{R}^{d}} \theta_{k} \circ \Psi d \mu_{t} & \leq \int_{\mathbb{R}^{d}} \Psi d \nu+\int_{0}^{t} \int_{\mathbb{R}^{d}}\left(\theta_{k}^{\prime} \circ \Psi\right) \Theta d \mu_{s} d s \\
+ & =\exp (M t) \int_{0}^{t} \exp (-M s)\left[\int_{\mathbb{R}^{d}} \Psi d \nu+\int_{0}^{s} \int_{\mathbb{R}^{d}}\left(\theta_{k}^{\prime} \circ \Psi\right) \Theta d \mu_{r} d r\right] d s .
\end{aligned}
$$

By Fatou's lemma we obtain the integrability of $\Psi$ with respect to $\mu_{t}$ and finally arrive at the desired estimates.

If the functions $t \mapsto \int \zeta d \mu_{t}$, where $\zeta \in C_{0}^{\infty}\left(\mathbb{R}^{d}\right)$, are continuous on $[0,1]$, then such functions are continuous also with $\zeta \in C_{b}\left(\mathbb{R}^{d}\right)$, which yields that our estimates are pointwise. Indeed, the right-hand side $R(t)$ of the first inequality in (2.3) is continuous in $t$. Let $\Psi_{n}:=\min (\Psi, n)$ and let $J_{n}(t)$ and $J(t)$ be the integrals of $\Psi_{n}$ and $\Psi$ against $\mu_{t}$, respectively. We have $J_{n}(t) \leq R(t)$ for a.e. $t$. By the continuity of both sides this inequality holds for all $t \in[0,1)$. By Fatou's theorem, one has $J(t) \leq R(t)$ also pointwise.

The reasoning is similar in the case of the open set $\Omega=\{\Psi<c\}$ in place of $\mathbb{R}^{d}$. Namely, we fix $\varepsilon>0$ and find a function $\theta \in C^{2}\left(\mathbb{R}^{1}\right)$ such that $\theta(r)=r$ if $r \leq c-\varepsilon$, $\theta(t)=c$ if $t \geq c+\varepsilon, 0 \leq \theta^{\prime} \leq 1$, and $\theta^{\prime \prime} \leq 0$. Then, letting $\zeta:=\theta \circ \Psi$, we obtain as above

$$
\int_{0}^{t} \int_{\Omega} \theta \circ \Psi d \mu_{s} d s \leq \exp (M t) \int_{0}^{t} \exp (-M s)\left[\int_{\Omega} \Psi d \nu+\int_{0}^{s} \int_{\Omega}\left(\theta^{\prime} \circ \Psi\right) \Theta d \mu_{r} d r\right] d s .
$$

Letting $\varepsilon \rightarrow 0$ we obtain our claim.

Corollary 2.3. Let $\mu=\left(\mu_{t}\right)_{t \in[0,1)}$ be a family of probability measures on $\mathbb{R}^{d}$ satisfying (1.1) and (1.3), where $\nu$ is a probability measure on $\mathbb{R}^{d}$. Let $\Psi \in C^{2}\left(\mathbb{R}^{d}\right)$ be a nonnegative function such that

$$
\lim _{|x| \rightarrow \infty} \Psi(x)=+\infty \quad \text { and } \quad L \Psi(t, x) \leq C+M \Psi(x) \quad \mu_{t} d t \text {-a.e. },
$$

where $C \geq 0$ and $M \geq 0$ are constants. Then one can find a nonnegative function $\Psi_{0} \in C^{2}\left(\mathbb{R}^{d}\right)$ such that

$$
\Psi_{0} \in L^{1}(\nu), \lim _{|x| \rightarrow \infty} \Psi_{0}(x)=+\infty \quad \text { and } \quad L \Psi_{0}(t, x) \leq C+M \quad \mu_{t} d t \text {-a.e. }
$$

Moreover, if $\mathfrak{M}$ is a uniformly tight family of probability measures on $\mathbb{R}^{d}$ and for every $\nu \in \mathfrak{M}$ there exists a solution $\mu^{\nu}=\left(\mu_{t}^{\nu}\right)_{t \in[0,1)}$ of the problem $L_{\nu}^{*} \mu^{\nu}=0, \mu_{0}^{\nu}=\nu$ 
in the sense of (1.1), (1.3) and for some nonnegative compact function $\Psi \in C^{2}\left(\mathbb{R}^{d}\right)$ one has

$$
L_{\nu} \Psi(t, x) \leq C+M \Psi(x) \quad \mu_{t}^{\nu} d t \text {-a.e. }
$$

then one can find a function $\Psi_{0}$ as above such that

$$
\sup _{\nu \in \mathfrak{M}} \operatorname{esssup} \int_{t \in[0,1)} \int_{\mathbb{R}^{d}} \Psi_{0} d \mu_{t}^{\nu} \leq C+M+\sup _{\nu \in \mathfrak{M}} \int_{\mathbb{R}^{d}} \Psi_{0} d \nu<\infty .
$$

If the functions $t \mapsto \int \zeta d \mu_{t}$, where $\zeta \in C_{0}^{\infty}\left(\mathbb{R}^{d}\right)$, are continuous on $[0,1]$, then esssup can be replaced by sup. The same is true in the case where $\mathbb{R}^{d}$ is replaced by a bounded open set.

Proof. Indeed, one can find a function $\theta \in C^{2}(\mathbb{R})$ such that $\theta(0)=0, \theta$ is nonnegative on $\mathbb{R}_{+}, \Psi_{1}:=\theta \circ \Psi \in L^{1}(\nu), \lim _{r \rightarrow+\infty} \theta(r)=+\infty, 0 \leq \theta^{\prime} \leq 1$, and $\theta^{\prime \prime} \leq 0$. To this end, it suffices to find a function $\theta$ with the listed properties such that it is integrable with respect to the measure $\sigma=\nu \circ \Psi^{-1}$. We find increasing numbers $T_{k}$ such that $T_{k+1}-T_{k} \geq T_{k}-T_{k-1} \geq 1$ and $\sigma\left(\left[T_{k}, \infty\right)\right) \leq 2^{-k}$. Let $\theta\left(T_{k}\right)=k-1$. Interpolating between $T_{k}$ and $T_{k+1}$ we obtain a $\sigma$-integrable increasing concave function $\theta_{0}$, which is not $C^{2}$, however. But now we take for $\theta(t)$ the integral over $[0, t]$ of a smooth function $g$ chosen as follows: $g(t)=\theta_{0}^{\prime}(t)$ if $t \in\left(T_{k}, T_{k+1}-k^{-1}\right), g^{\prime}(t) \leq 0$. It is readily seen that we obtain a required function. Then

$$
L \Psi_{1} \leq \theta^{\prime}(\Psi) L \Psi \leq \theta^{\prime}(\Psi)(C+M \Psi) \leq C+M \theta(\Psi)=C+M \Psi_{1}
$$

because $\theta^{\prime}(\Psi) \Psi \leq \theta(\Psi)$ as explained above. Replacing $\Psi_{1}$ by $\Psi_{0}:=\log \left(\Psi_{1}+1\right)$, we find that

$$
L \Psi_{0}=\left(\Psi_{1}+1\right)^{-1} L \Psi_{1}-\left(\Psi_{1}+1\right)^{-2}\left\langle A \nabla \Psi_{1}, \nabla \Psi_{1}\right\rangle \leq C+M .
$$

Moreover, for any uniformly tight family of probability measures $\mathfrak{M}$, such a function $\theta$ can be found with the property that

$$
\sup _{\nu \in \mathfrak{M}} \int_{\mathbb{R}^{d}} \Psi_{0} d \nu<\infty
$$

This is seen from the above reasoning. Now we apply (2.4) with $K=C+M$. If the functions $t \mapsto \int \zeta d \mu_{t}$, where $\zeta \in C_{0}^{\infty}\left(\mathbb{R}^{d}\right)$, are continuous on $[0,1]$, then esssup can be replaced by sup because (2.4) holds pointwise in this case.

Remark 2.4. It is obvious from the proof that the condition $L \Psi \leq C$ in Lemma 2.2 can be relaxed as follows: there exists a measurable set $E \subset \mathbb{R}^{d}$ such that $L \Psi(t, x) \leq C+C \Psi(x) \quad \mu_{t} d t$-a.e. on $(0,1) \times\left(\mathbb{R}^{d} \backslash E\right)$ and

$$
C^{\prime}:=\int_{0}^{1} \int_{E}|L \Psi(t, x)| \mu_{t}(d x) d t<\infty .
$$

Then in the left-hand side of (2.4) one should add $C^{\prime}$.

It is also worth mentioning that if in the situation of Lemma 2.2 the functions $a^{i j}$ are bounded on bounded subsets of $[0,1] \times \mathbb{R}^{d}$ and (2.2) holds only for $x$ outside some bounded set, then one can find another nonnegative function $\Psi_{0} \in C^{2}\left(\mathbb{R}^{d}\right)$ such that $\Psi_{0}$ coincides with $\Psi$ outside some bounded set and $L \Psi_{0} \leq C_{0} \mu_{t} d t$-a.e., where $C_{0}$ is a positive constant. Indeed, let $\theta \in C^{\infty}\left(\mathbb{R}^{1}\right)$ be such that $\theta(s)=0$ if $s \leq-1, \theta(s)=s$ if $s \geq 1,0 \leq \theta^{\prime} \leq 1$. There exists $k$ such that $L \Psi \leq C \mu_{t} d t$-a.e. if $|x| \geq k$. Let

$$
M:=\sup _{s}\left|\theta^{\prime \prime}(s)\right| \sup _{t \in[0,1],|x| \leq k+2}\langle A(t, x) \nabla \Psi(x), \nabla \Psi(x)\rangle, \quad \Psi_{0}(x):=\theta(\Psi(x)-k) .
$$


Then

$$
\begin{aligned}
L \Psi_{0}(t, x) & =\theta^{\prime}(\Psi(x)-k-1) L \Psi(t, x)+\theta^{\prime \prime}(\Psi(x)-k-1)\langle A(t, x) \nabla \Psi(x), \nabla \Psi(x)\rangle \\
& \leq C+M,
\end{aligned}
$$

since $\theta^{\prime}(\Psi(x)-k-1)=0$ if $|x| \leq k, L \Psi(x) \leq C$ if $|x| \geq k$, and $\theta^{\prime \prime}(\Psi(x)-k-1)=0$ if $|x| \leq k+2$.

Let us consider examples of how (2.2) can be verified in terms of the coefficients of $L$.

Example 2.5. (i) Suppose that

$$
\left|a^{i j}(t, x)\right| \leq c_{1}+c_{2}|x|^{2}, \quad\langle b(t, x), x\rangle \leq c_{3}+c_{4}|x|^{2}
$$

for some constants $c_{i}$. Then, letting $\Psi(x):=|x|^{2 k}, k>0$, we obtain $L \Psi \leq C+C \Psi$ for a sufficiently large $C>0$. Consequently, if a solution $\mu$ exists and $|x|^{2 k} \in L^{1}\left(\mu_{0}\right)$, then, for a.e. $t$, we have

$$
\int_{\mathbb{R}^{d}}|x|^{2 k} \mu_{t}(d x) \leq e^{C} \int_{\mathbb{R}^{d}}|x|^{2 k} \mu_{0}(d x)+C e^{C} .
$$

(ii) Suppose that

$$
\left|a^{i j}(t, x)\right| \leq c_{1}+c_{2} \ln \left(|x|^{2}+1\right), \quad\langle b(t, x), x\rangle \leq c_{3}+c_{4}|x|^{2}+c_{5}|x|^{2} \ln \left(|x|^{2}+1\right),
$$

for some constants $c_{i}$. Then, letting $\Psi(x):=\ln \left(|x|^{2}+1\right)$, we find

$$
\begin{gathered}
\partial_{x_{i}} \Psi(x)=2 x_{i}\left(|x|^{2}+1\right)^{-1}, \\
\partial_{x_{j}} \partial_{x_{i}} \Psi(x)=2 \delta_{i j}-4 x_{i} x_{j}\left(|x|^{2}+1\right)^{-2},
\end{gathered}
$$

which yields

$$
\begin{aligned}
L \Psi(t, x) & =2 \operatorname{trace} A(t, x)-4\left(|x|^{2}+1\right)^{-2}\langle A(t, x) x, x\rangle+2\left(|x|^{2}+1\right)^{-1}\langle b(t, x), x\rangle \\
& \leq C+C \Psi(x)
\end{aligned}
$$

for a sufficiently large $C>0$. Consequently, if a solution $\mu$ exists and $\ln \left(|x|^{2}+1\right) \in$ $L^{1}\left(\mu_{0}\right)$, then, for a.e. $t$, we have

$$
\int_{\mathbb{R}^{d}} \ln \left(|x|^{2}+1\right) \mu_{t}(d x) \leq e^{C} \int_{\mathbb{R}^{d}} \ln \left(|x|^{2}+1\right) \mu_{0}(d x)+C e^{C} .
$$

Moreover, letting $\Psi(x)=\left|\ln \left(|x|^{2}+1\right)\right|^{2}$, we also have $L \Psi \leq C+C \Psi$, hence

$$
\int_{\mathbb{R}^{d}}\left|\ln \left(|x|^{2}+1\right)\right|^{2} \mu_{t}(d x) \leq e^{C} \int_{\mathbb{R}^{d}}\left|\ln \left(|x|^{2}+1\right)\right|^{2} \mu_{0}(d x)+C e^{C} .
$$

(iii) Suppose that

$$
\langle A(t, x) x, x\rangle \leq \gamma_{1}+\alpha|x|^{2 \beta}, \quad\langle b(t, x), x\rangle \leq \gamma_{2}-(2 \alpha c k+\varepsilon)|x|^{2 k+2 \beta-2}
$$

with some positive constants $\gamma_{1}, \gamma_{2}, \alpha, \beta, c, k, \varepsilon$. Let $\Psi(x)=\exp \left(c|x|^{2 k}\right)$. Then

$$
\begin{aligned}
L \Psi(t, x) & =2 c k \text { trace } A(t, x)|x|^{2 k-2} \Psi(x)+4 c k(k-1)\langle A(t, x) x, x\rangle|x|^{2 k-4} \Psi(x) \\
& +(2 c k)^{2}\langle A(t, x) x, x\rangle|x|^{4 k-4} \Psi(x)+2 c k|x|^{2 k-2} \Psi(x)\langle b(t, x), x\rangle \\
& \leq c_{0}-\varepsilon|x|^{2 k+2 \beta-2} \Psi(x)
\end{aligned}
$$

with some constant $c_{0}$. Hence, if $\beta \geq 1$ and $\Psi \in L^{1}\left(\mu_{0}\right)$, then

$$
\operatorname{esssup}_{t \in[0,1)} \int_{\mathbb{R}^{d}} \exp \left(c|x|^{2 k}\right) \mu_{t}(d x)<\infty \text {. }
$$


Let us introduce the following conditions on $A, b, p \in[1,+\infty)$, and an open ball $B \subset \mathbb{R}^{d}:$

(C1) there exist two constants $M_{1}=M_{1}(B)$ and $M_{2}=M_{2}(B)$ such that for all $i, j$ one has

$$
\operatorname{det}_{(t, x) \in[0,1] \times B} A(t, x) \geq M_{1} \text { and } \sup _{t \in[0,1]}\left\|a^{i j}(t, \cdot)\right\|_{H^{p, 1}(B)} \leq M_{2} .
$$

(C2) there exists $M_{3}=M_{3}(B)$ such that for all $i$ one has

$$
\sup _{t \in[0,1]}\left\|b^{i}(t, \cdot)\right\|_{L^{p}(B)} \leq M_{3} .
$$

It follows from (C1) and the Sobolev embedding theorem that if $p>d$, then every function $a^{i j}$ has a jointly measurable version such that all functions $x \mapsto a^{i j}(t, x)$, $t \in(0,1)$, are Hölder continuous of order $1-d / p$ and bounded on $B$ uniformly in $t$ (their Hölder and sup-norms on $B$ are estimated by a constant depending on $p, d$, $B$ and $M_{2}$ ). Below we use the same notation $a^{i j}$ for these particular versions.

The main result of this work is based on the following lemma.

Set

$$
b_{0}:=A^{-1 / 2}(b-\Gamma), \quad \Gamma:=\left(\Gamma^{1}, \ldots, \Gamma^{d}\right), \quad \Gamma^{j}=\partial_{x_{i}} a^{i j} .
$$

Lemma 2.6. Let $\Omega$ be an open bounded set in $\mathbb{R}^{d}$ with a smooth boundary and volume $|\Omega|$, let the functions $a^{i j}$ and $b^{i}$ be infinitely differentiable in the second argument on $(0,1) \times \Omega_{1}$, where $\Omega_{1} \supset \bar{\Omega}$ is a bounded open set, and let $A(t, x) \geq \alpha I$ for some number $\alpha>0$. Suppose that $\mu=\varrho(t, x) d x d t$, where every $\varrho(t, \cdot), t>0$, is a twice continuously differentiable positive probability density on $\Omega$ such that

$$
\frac{\partial \varrho(t, x)}{\partial t}=\partial_{x_{i}} \partial_{x_{j}}\left(a^{i j} \varrho\right)-\partial_{x_{i}}\left(b^{i} \varrho\right)
$$

in $(0,1) \times \Omega$. Suppose also that $\varrho$ is continuous on the closure of $[0,1] \times \Omega, \varrho(0, x)=\varrho_{0}$ with $\varrho_{0} \in C_{0}^{1}(\Omega)$, and

$$
\left\langle A \nabla \varrho(t, x)+[\Gamma(t, x)-b(t, x)] \varrho(t, x), \mathrm{n}_{\partial \Omega}(x)\right\rangle=0, \quad(t, x) \in[0,1] \times \partial \Omega,
$$

where $\mathrm{n}_{\partial \Omega}$ is the outer unit normal on $\partial \Omega$. Then

$$
\begin{aligned}
& \int_{0}^{1} \int_{\Omega}|\sqrt{A} \nabla \varrho(t, x)|^{2} \varrho(t, x)^{-1} d x d t \\
& \leq 2 \int_{0}^{1} \int_{\Omega}\left|b_{0}(t, x)\right|^{2} \varrho(t, x) d x d t+2 \int_{\Omega} \varrho_{0}(x) \log \varrho_{0}(x) d x+2|\Omega| .
\end{aligned}
$$

In addition, there is a constant $C(\Omega)$ such that

$$
\begin{aligned}
& \int_{0}^{1}\left(\int_{\Omega}|\varrho(t, x)|^{d /(d-2)} d x\right)^{(d-2) / d} d t \\
& \leq \frac{C(\Omega)}{\alpha}\left(\int_{0}^{1} \int_{\Omega}\left|b_{0}(t, x)\right|^{2} \varrho(t, x) d x d t+\int_{\Omega} \varrho_{0}(x) \log \varrho_{0}(x) d x+|\Omega|\right)+2|\Omega|^{(2 d-2) / d} .
\end{aligned}
$$

Finally, for every $p>d$, there is a constant $C(\Omega, p)$ such that

$$
\begin{aligned}
& \int_{0}^{1} \int_{\Omega}|\sqrt{A} \nabla \varrho(t, x)|^{2} \varrho(t, x)^{-1} d x d t \\
& \leq C(\Omega, p) \sup _{t \in(0,1)}\left(\int_{\Omega}\left|b_{0}(t, x)\right|^{p} d x\right)^{1 / p}+2 \int_{\Omega} \varrho_{0}(x) \log \varrho_{0}(x) d x+C(\Omega, p) .
\end{aligned}
$$


Proof. It suffices to consider the case $p=d$. Let us multiply both sides of (2.5) by $\log \varrho$ and integrate over $[0,1] \times \Omega$. Applying the integration by parts formula, condition (2.6) and the identity $\int_{\Omega} \varrho(t, x) d x=\int_{\Omega} \varrho_{0}(x) d x=1$, we obtain

$$
\begin{aligned}
\int_{\Omega} \varrho(1, x) \log \varrho(1, x) & d x-\int_{\Omega} \varrho_{0}(x) \log \varrho_{0}(x) d x \\
= & -\int_{0}^{1} \int_{\Omega} \frac{a^{i j} \partial_{x_{i}} \varrho \partial_{x_{j}} \varrho}{\varrho} d x d t+\int_{0}^{1} \int_{\Omega} \frac{\partial_{x_{j}} \varrho}{\varrho}\left(b^{j}-\partial_{x_{i}} a^{i j}\right) \varrho d x d t .
\end{aligned}
$$

Let $E:=\int_{\Omega} \varrho_{0} \log \varrho_{0} d x$. Note that $\varrho(1, x) \log \varrho(1, x) \geq-1$. Therefore,

$$
J:=\int_{0}^{1} \int_{\Omega}\langle A \nabla \varrho, \nabla \varrho\rangle \frac{1}{\varrho} d x d t \leq \int_{0}^{1} \int_{\Omega}\left\langle A^{1 / 2} \frac{\nabla \varrho}{\sqrt{\varrho}}, b_{0}\right\rangle \sqrt{\varrho} d x d t+E+|\Omega| .
$$

Set

$$
\left\|b_{0}\right\|_{2, \mu}:=\left(\int_{0}^{1} \int_{\Omega}\left|b_{0}\right|^{2} \varrho(t, x) d x d t\right)^{1 / 2} .
$$

Finally, let $\left\|b_{0}\right\|_{p}$ denote the norm of $\left|b_{0}\right|$ in $L^{p}([0,1] \times \Omega)$ with respect to Lebesgue measure. By the Cauchy inequality we find

$$
J \leq \sqrt{J}\left\|b_{0}\right\|_{2, \mu}+E+|\Omega|,
$$

which yields

$$
\sqrt{J} \leq\left(\left\|b_{0}\right\|_{2, \mu}^{2} / 4+E+|\Omega|\right)^{1 / 2}+\left\|b_{0}\right\|_{2, \mu} / 2 \leq\left\|b_{0}\right\|_{2, \mu}+\sqrt{E+|\Omega|} .
$$

Let $\alpha(t)$ denote the integral of $\sqrt{\varrho(t, x)}$ over $\Omega$. By the Sobolev inequality, there is a constant $C(\Omega)$ such that

$$
\left(\int_{\Omega}|\sqrt{\varrho(t, x)}-\alpha(t)|^{2 d /(d-2)} d x\right)^{(d-2) / d} \leq C(\Omega) \int_{\Omega}|\nabla \sqrt{\varrho}|^{2} d x .
$$

Noting that $\alpha(t) \leq \sqrt{|\Omega|}$, we arrive at the estimate

$$
\left(\int_{\Omega} \varrho(t, x)^{d /(d-2)} d x\right)^{(d-2) / 2 d} \leq \frac{1}{2}\left(C(\Omega) \int_{\Omega}|\nabla \varrho|^{2} \varrho^{-1} d x\right)^{1 / 2}+|\Omega|^{(d-1) / d} .
$$

Finally we obtain

$$
\left(\int_{\Omega} \varrho(t, x)^{d /(d-2)} d x\right)^{(d-2) / d} \leq \frac{1}{2} C(\Omega) \int_{\Omega}|\nabla \varrho|^{2} \varrho^{-1} d x+2|\Omega|^{(2 d-2) / d},
$$

which gives $(2.8)$.

In order to prove the last claim, we observe that, letting $q=2 p /(p-2)$, for every $\varepsilon>0$, one can find a constant $C(\Omega, p, \varepsilon)$ such that

$$
\left(\int_{\Omega} \varphi(x)^{q} d x\right)^{1 / q} \leq \varepsilon\left(\int_{\Omega}|\nabla \varphi(x)|^{2} d x\right)^{1 / 2}+C(\Omega, p, \varepsilon)
$$

for every $\varphi \in W^{2,1}(\Omega)$ with $\|\varphi\|_{L^{2}(\Omega)} \leq 1$. Indeed, the Sobolev inequality gives an estimate $\|\varphi\|_{L^{q}(\Omega)} \leq C\|\mid \nabla \varphi\|_{L^{2}(\Omega)}+C$. If our claim is not true, then there is a sequence of functions $\varphi_{n} \in W^{2,1}(\Omega)$ with $\|\varphi\|_{L^{2}(\Omega)}=1$ and $\|\varphi\|_{L^{q}(\Omega)} \geq \varepsilon\|\mid \nabla \varphi\|_{L^{2}(\Omega)}+n$. It follows by the aforementioned estimate that $c_{n}:=\|\mid \nabla \varphi\|_{L^{2}(\Omega)} \rightarrow \infty$. Hence $\psi_{n}:=\varphi_{n} / c_{n} \rightarrow 0$ in $L^{2}(\Omega)$ and $\left\|\psi_{n}\right\|_{q} \geq \varepsilon$. Let us take $r \in(q, 2 d /(d-2))$. The embedding of $W^{2,1}(\Omega) \rightarrow L^{r}(\Omega)$ is compact, hence $\left\{\psi_{n}\right\}$ contains a subsequence 
convergent in $L^{r}(\Omega)$. Clearly, this subsequence must converge in $L^{r}(\Omega)$ to zero, as it converges to zero in $L^{2}(\Omega)$. This contradicts the fact that $\left\|\psi_{n}\right\|_{q} \geq \varepsilon$ and proves our claim. Now it remains to use the estimate

$$
\begin{aligned}
\left\|b_{0}\right\|_{2, \mu}^{2} & \leq \int_{0}^{1}\left(\int_{\Omega}\left|b_{0}(t, x)\right|^{p} d x\right)^{2 / p}\left(\int_{\Omega} \varrho(t, x)^{q} d x\right)^{1 / q} d t \\
& \leq \sup _{t \in(0,1)}\left\|b_{0}(t, \cdot)\right\|_{L^{p}(\Omega)}[\varepsilon J+C(\Omega, p, \varepsilon)]
\end{aligned}
$$

and then choose $\varepsilon<4^{-1}\left[\sup _{t \in(0,1)}\left\|b_{0}(t, \cdot)\right\|_{L^{p}(\Omega)}+1\right]^{-1}$.

Corollary 2.7. Suppose that in Lemma 2.6 there exist a nonnegative compact function $\Psi \in C^{2}\left(\mathbb{R}^{d}\right)$ and a constant $M$ such that

$$
L \Psi(t, x) \leq M+M \Psi(x) \text { and }\left|b_{0}(t, x)\right|^{2} \leq \Psi(x) .
$$

Then we have

$$
\begin{aligned}
& \int_{0}^{1} \int_{\Omega}|\sqrt{A} \nabla \varrho(t, x)|^{2} \varrho(t, x)^{-1} d x d t \\
\leq & 2\left(M e^{M}+1\right) \int_{\Omega} \Psi(x) \varrho_{0}(x) d x+2 M\left(M e^{M}+1\right)+2 \int_{\Omega} \varrho_{0}(x) \log \varrho_{0}(x) d x+2|\Omega| .
\end{aligned}
$$

In particular, this is true for $\Psi(x)=|x|^{2 k}$ with $k \geq 1$ provided that

$$
\operatorname{trace} A(t, x) \leq C+C|x|^{2}, \quad\left|b_{0}(t, x)\right|^{2} \leq C+C|x|^{2 k}, \quad\langle b(t, x), x\rangle \leq C+C|x|^{2} .
$$

Proof. It suffices to use the version of (2.3) for $\Omega$ mentioned in Lemma 2.2 in order to estimate the integral of $\left|b_{0}\right|^{2} \leq \Psi$ over $[0,1) \times \Omega$.

It is worth noting that under some additional assumptions on $A$ and $b$, much stronger global bounds of a similar type are established in [7].

\section{Main Results}

Theorem 3.1. Let $p>d+2$ and let $A$ and $b$ satisfy $(\mathrm{C} 1)$ and $(\mathrm{C} 2)$ for every ball. Assume that there exists a nonnegative compact function $\Psi \in C^{2}\left(\mathbb{R}^{d}\right)$ and a constant $C \geq 0$ such that

$$
L \Psi(t, x) \leq C+C \Psi(x) \text { a.e. in }(0,1) \times \mathbb{R}^{d} .
$$

Then, for every probability measure $\nu$, there exists a family $\mu=\left(\mu_{t}\right)_{t \in[0,1)}$ of probability measures on $\mathbb{R}^{d}$ satisfying (1.1) and (1.3) such that $t \mapsto \int_{\mathbb{R}^{d}} \zeta d \mu_{t}$ is continuous on $[0,1)$ for every $\zeta \in C_{0}^{\infty}\left(\mathbb{R}^{d}\right)$.

Proof. Step 1. Let us fix a probability density $\varrho_{0} \in C_{0}^{\infty}\left(\mathbb{R}^{d}\right)$ and set $\nu=\varrho_{0} d x$. Let $\Omega$ be a bounded connected open set in $\mathbb{R}^{d}$ having a smooth boundary and containing the support of $\zeta$. We prove that there is a Borel function $\varrho_{\Omega}$ on $[0,1) \times \Omega$ such that every $\varrho_{\Omega}(t, \cdot)$ is a probability density on $\Omega$ and $\mu_{\Omega}:=\varrho_{\Omega} d x d t$ satisfies (1.1) and (1.3) on $[0,1) \times \Omega$. First we consider the case when $A$ and $b$ are smooth in $x$ and there exists a compact set $K \subset \Omega$ such that, letting $\Gamma=\left(\Gamma^{1}, \ldots, \Gamma^{d}\right), \Gamma^{j}:=\partial_{x_{i}} a^{i j}$, one has $b(t, x)-\Gamma(t, x)=0$ if $x \notin K$. It is known that there is a nonnegative continuous solution $\varrho_{\Omega} \in L^{p}\left([0,1], W^{p, 1}(\Omega)\right)$ of the initial value problem

$$
\frac{\partial \varrho_{\Omega}}{\partial t}=\partial_{x_{i}} \partial_{x_{j}}\left(a^{i j} \varrho_{\Omega}\right)-\partial_{x_{i}}\left(b^{i} \varrho_{\Omega}\right), \quad \varrho_{\Omega}(0, x)=\varrho_{0}(x),
$$


with the boundary condition $\left.\left\langle\nabla \varrho_{\Omega}, A \mathrm{n}_{\partial \Omega}\right\rangle\right|_{\partial \Omega}=0$, where $\mathrm{n}_{\partial \Omega}$ is the outer unit normal on $\partial \Omega$ (see [13]). In addition, one has

$$
\int_{\Omega} \varrho_{\Omega}(t, x) d x=1
$$

for all $t \in[0,1)$ because one has

$$
\int_{\Omega}\left[\partial_{x_{i}} \partial_{x_{j}}\left(a^{i j} \varrho_{\Omega}\right)-\partial_{x_{i}}\left(b^{i} \varrho_{\Omega}\right)\right] d x=\int_{\Omega} \operatorname{div}\left(A \nabla \varrho_{\Omega}+\varrho_{\Omega} \Gamma-\varrho_{\Omega} b\right) d x=0
$$

by the integration by parts formula and the equalities $\left.\left\langle\nabla \varrho_{\Omega}, A \mathrm{n}_{\partial \Omega}\right\rangle\right|_{\partial \Omega}=0$ and $\left\langle b-\Gamma, \mathrm{n}_{\partial \Omega}\right\rangle=0$.

Step 2. We shall obtain a solution on $[0,1) \times \mathbb{R}^{d}$ as a limit of solutions $\varrho_{n}$ with smooth compactly supported initial distributions. In the general case, one can find connected open sets $\Omega_{n}$ with smooth boundaries such that

$$
\{\Psi \leq n\} \subset \Omega_{n} \subset\{\Psi<n+1\} .
$$

Let $\varrho_{n, 0} \in C_{0}^{\infty}\left(\Omega_{n}\right)$ be probability densities such that the measures $\nu_{n}=\varrho_{n, 0} d x$ converge weakly to $\nu$ and the integrals $\int \Psi d \nu_{n}$ converge to $\int \Psi d \nu$.

We can find Borel functions $a_{n}^{i j}$ and $b_{n}^{j}$ that are smooth in $x$ and possess the following properties: for every fixed bounded domain $\Omega$ in $\mathbb{R}^{d}$

1) the functions $a_{n}^{i j}$ are uniformly bounded on $[0,1] \times \Omega, a_{n}^{i j}(t, \cdot) \rightarrow a^{i j}(t, \cdot)$ in $W^{p, 1}(\Omega)$ for every $t$ and $\sup _{t}\left\|a_{n}^{i j}(t, \cdot)\right\|_{W^{p, 1}(\Omega)}<\infty$, the matrices $A_{n}:=\left(a_{n}^{i j}\right)$ are positive symmetric and $\inf _{(t, x) \in[0,1] \times \Omega} \operatorname{det} A_{n}(t, x)>0$,

2) $b_{n}(t, \cdot) \rightarrow b(t, \cdot)$ in $L^{p}(\Omega)$ for every $t$ and $\sup _{n} \sup _{t}\left\|b_{n}(t, \cdot)\right\|_{L^{p}(\Omega)}<\infty$,

3) for each $n$, there exists a compact set $K_{n} \subset \Omega_{n}$ such that

$$
b_{n}^{j}(t, x)-\partial_{x_{i}} a_{n}^{i j}(t, x)=0 \quad \text { if } x \notin K_{n} .
$$

For every $n$ we have a solution $\varrho_{n}=\varrho_{\Omega, n}$ of (3.2) on $\Omega_{n}$ with the coefficients $A_{n}$ and $b_{n}$. Clearly, condition (2.6) is fulfilled with $\varrho_{n}$ and $\Omega_{n}$ in place of $\varrho$ and $\Omega$. It follows from Lemma 2.6 that we can find a subsequence in this sequence that converges almost everywhere on $(0,1) \times \mathbb{R}^{d}$ to a function $\varrho_{\Omega}$. Our conditions on $a_{n}^{i j}$ and $b_{n}^{j}$ imply on account of results in [3, Section 3] that this convergence is uniform on compact subsets in $(0,1) \times \mathbb{R}^{d}$ and that $\varrho_{\Omega}$ satisfies the required equation.

Now me make use of the Lyapunov function $\Psi$. We have to show that $\varrho(t, \cdot)$ is a probability density and that (1.3) is fulfilled. According to Corollary 2.3 we may assume that $L \Psi \leq 2 C$, which ensures that for every $t$, the sequence of probability measures $\varrho_{n}(t, x) d x$ is uniformly tight on $\mathbb{R}^{d}$. Hence every $\varrho(t, \cdot)$ is a probability density. The only nontrivial thing is to justify (1.3). Let $\zeta \in C_{0}^{\infty}\left(\mathbb{R}^{d}\right)$. We set

$$
h_{n}(t):=\int_{\Omega_{n}} \zeta(x) \varrho_{n}(t, x) d x
$$

It suffices to show that uniformly in $n$ one has

$$
\lim _{t \rightarrow 0} h_{n}(t)=\int_{\mathbb{R}^{d}} \zeta(x) \nu(d x)
$$

Let $w_{n}^{\tau}$ be the solution of the backward Cauchy problem

$$
\partial w_{n}^{\tau} / \partial t+a_{n}^{i j} \partial_{x_{i}} \partial_{x_{j}} w_{n}^{\tau}+b_{n}^{j} \partial_{x_{j}} w_{n}^{\tau}=0, \quad w_{n}^{\tau}(\tau, x)=\zeta(x), \quad(t, x) \in[0, \tau] \times \Omega_{n},
$$

with Dirichlet boundary condition. Multiplying the equation for $\varrho_{n}$ by $w_{n}^{\tau}$ and integrating over $[0, \tau] \times \Omega_{n}$ we obtain

$$
\int_{\Omega_{n}} w_{n}^{\tau}(0, x) \varrho_{n, 0}(x) d x=\int_{\Omega_{n}} \zeta(x) \varrho_{n}(\tau, x) d x=h_{n}(\tau) .
$$


Indeed, on account of the integration by parts formula and boundary conditions for $w_{n}^{\tau}$ and $\varrho_{n}$ we have

$$
\begin{gathered}
0=\int_{0}^{\tau} \int_{\Omega_{n}} w_{n}^{\tau}\left[\frac{\partial \varrho_{n}}{\partial t}-\partial_{x_{i}} \partial_{x_{j}}\left(a_{n}^{i j} \varrho_{n}\right)+\partial_{x_{i}}\left(b_{n}^{i} \varrho_{n}\right)\right] d x d t \\
=\int_{\Omega_{n}}\left[w_{n}^{\tau}(0, x) \varrho_{n, 0}(x)-\zeta(x) \varrho_{n}(\tau, x)\right] d x-\int_{0}^{\tau} \int_{\Omega_{n}} \varrho_{n} \frac{\partial w_{n}^{\tau}}{\partial t} d x d t \\
\quad+\int_{0}^{\tau} \int_{\Omega_{n}} \partial_{x_{i}} w_{n}^{\tau}\left[\partial_{x_{j}}\left(a_{n}^{i j} \partial_{x_{j}} \varrho_{n}\right)-b_{n}^{i} \varrho_{n}\right] d x d t \\
=\int_{\Omega_{n}}\left[w_{n}^{\tau}(0, x) \varrho_{n, 0}(x)-\zeta(x) \varrho_{n}(\tau, x)\right] d x \\
-\int_{0}^{\tau} \int_{\Omega_{n}} \varrho_{n} \frac{\partial w_{n}^{\tau}}{\partial t} d x d t-\int_{0}^{\tau} \int_{\Omega_{n}}\left[a_{n}^{i j} \partial_{x_{j}} \partial_{x_{i}} w_{n}^{\tau}+b_{n}^{i} \partial_{x_{i}} w_{n}^{\tau}\right] \varrho_{n} d x d t \\
=\int_{\Omega_{n}}\left[w_{n}^{\tau}(0, x) \varrho_{n, 0}(x)-\zeta(x) \varrho_{n}(\tau, x)\right] d x .
\end{gathered}
$$

Given $\delta>0$, one can find a compact set $K \subset \mathbb{R}^{d}$ such that $\nu_{n}(K) \geq 1-\delta$ for all $n$. We may assume that $K$ contains the support of $\zeta$. In addition, it is readily seen that the functions $w_{n}^{\tau}$ are uniformly bounded. Moreover, one has $\lim _{\tau \rightarrow 0} w_{n}^{\tau}(0, x) \rightarrow \zeta(x)$ uniformly in $n \in \mathbb{N}$ and $x \in K$, see [12, Ch. III, Theorem 7.1 and Theorem 10.1] or [11]. Now by weak convergence of $\nu_{n}$ to $\nu$ and (3.3) we complete the proof.

Remark 3.2. (i) It is clear from the proof of the above theorem that in the case when the functions $b^{i}$ are bounded on bounded subsets of $(0,1) \times \mathbb{R}^{d}$, the nondegeneracy condition on $A$ can be slightly relaxed as follows: it suffices to have

$$
\inf _{(t, x) \in\left[\tau_{1}, \tau_{2}\right] \times K} \operatorname{det} A(t, x)>0
$$

for every $\left[\tau_{1}, \tau_{2}\right] \subset(0,1)$ and every compact set $K \subset \mathbb{R}^{d}$.

(ii) It follows from Remark 2.4 and the above proof that condition (3.1) can be relaxed as follows: there exists a compact set $K \subset \mathbb{R}^{d}$ such that $L \Psi(t, x) \leq C$ a.e. in $(0,1) \times\left(\mathbb{R}^{d} \backslash K\right)$.

(iii) It is also clear that the solution constructed above has the following property: for a.e. $t$, the measure $\mu_{t}$ has a density from the Sobolev class $H_{l o c}^{p, 1}\left(\mathbb{R}^{d}\right)$. As shown in [3], this is true for any solution of (1.1) under our local assumptions on $A$ and $b$. Hence, under these assumptions, equation (1.1) can be written in the classical weak form after integrating by parts in the term with $\partial_{x_{i}} \partial_{x_{j}} u$. Below we consider more general equations whose solutions do not have such a property.

Corollary 3.3. Suppose that there is a constant $C$ such that

$$
\|A(t, x)\| \leq C+C \ln \left(|x|^{2}+1\right), \quad(t, x) \in[0,1) \times \mathbb{R}^{d},
$$

and, for every compact set $K \subset \mathbb{R}^{d}$ and every $\left[\tau_{1}, \tau_{2}\right] \subset(0,1)$, one has

$$
\inf _{(t, x) \in\left[\tau_{1}, \tau_{2}\right] \times K} \operatorname{det} A(t, x)>0, \sup _{(t, x) \in[0,1) \times K}|b(t, x)|<\infty .
$$

Assume also that there is a constant $M$ such that

$$
\langle b(t, x), x\rangle \leq M\left(1+|x|^{2}\right) \ln \left(|x|^{2}+1\right), \quad(t, x) \in[0,1) \times \mathbb{R}^{d} .
$$

Then, for every probability measure $\nu$ on $\mathbb{R}^{d}$, there exists a family $\left(\mu_{t}\right)_{t \in[0,1)}$ of probability measures on $\mathbb{R}^{d}$ satisfying (1.1) and (1.3) such that $t \mapsto \int_{\mathbb{R}^{d}} \zeta d \mu_{t}$ is continuous 
on $[0,1)$ for every $\zeta \in C_{0}^{\infty}\left(\mathbb{R}^{d}\right)$. The same is true if we replace (3.4) and (3.5) by

$$
\|A(t, x)\| \leq C+C|x|^{2}, \quad\langle b(t, x), x\rangle \leq C+C|x|^{2}, \quad(t, x) \in[0,1) \times \mathbb{R}^{d} .
$$

If the functions $b^{i}$ and $a^{i j}$ are continuous in $x$ for a.e. fixed $t$, then the same is true without the assumption that $\operatorname{det} A$ is strictly positive.

Proof. As in [1], we find Borel measurable mappings $A_{n}$ on $(0,1) \times \mathbb{R}^{d}$ with values in the space of nonnegative symmetric operators such that the functions $a_{n}^{i j}(t, x)$ are smooth in $x$ and satisfy the following conditions: $a_{n}^{i j} \rightarrow a^{i j}$ a.e., $A_{n}(t, x) \geq n^{-1} \cdot \mathrm{I}$ for every $n$,

$$
\sup _{n} \sup _{t, x}\left|\ln \left(|x|^{2}+1\right)\right|^{-1}\left(|x|^{2}+1\right)^{-1}\left\|A_{n}(t, x)\right\|<\infty,
$$

for every ball $B \subset \mathbb{R}^{d}$ and every closed interval $\left[\tau_{1}, \tau_{2}\right] \subset(0,1)$, one has

$$
\sup _{n} \sup _{(t, x) \in\left[\tau_{1}, \tau_{2}\right] \times B}\left\|A_{n}(t, x)^{-1}\right\|<\infty .
$$

One can find $A_{n}$ of the form $A_{n}=n^{-1} \cdot \mathrm{I}+A * \theta_{n}$, where $\theta_{n}(x)=n^{d} \theta(n x)$ with some smooth compactly supported probability density $\theta$. Let $L_{n}$ denote the operator with the diffusion matrix $A_{n}$ and drift $b$. Letting $\Psi(x)=\ln \left(|x|^{2}+1\right)$, by the calculations from Example 2.5(ii) we have for all $n$ and some constant $C_{1}$

$$
L_{n} \Psi(t, x) \leq C_{1}+C_{1}|x|^{2} \ln \left(|x|^{2}+1\right) .
$$

For every $n$ we obtain a solution $\mu^{n}$ with a density $\varrho_{n}$ for the equation with $L_{n}$. By Corollary 2.3 the sequence $\left\{\mu^{n}\right\}$ is uniformly tight on $[0,1] \times \mathbb{R}^{d}$. Hence we can choose a weakly convergent subsequence. Let $\mu$ denote its limit. Moreover, according to [3], we can pick a further subsequence (denoted again by $\mu^{n}$ ) for which the densities $\varrho_{n}$ converge locally uniformly. Then, similarly to [1], we verify that $\mu$ satisfies (1.2). Finally, (1.3) follows by the fact that for every function $\zeta \in C_{0}^{\infty}\left(\mathbb{R}^{d}\right)$, the functions

$$
t \mapsto \int_{\mathbb{R}^{d}} \zeta d \mu_{t}^{n}
$$

are uniformly Lipschitzian on $[0,1 / 2]$, which is clear from $(2.1)$ and the uniform boundedness of $A$ and $b$ on $[0,1] \times \operatorname{supp}, \zeta$.

A more general result is valid if $A$ and $b$ are continuous in $x$. A justification is similar to the previous corollary (see also the proof of the analogous but a bit weaker corollary 3.4 in [1]).

Corollary 3.4. Suppose that the functions $x \mapsto a^{i j}(t, x)$ and $x \mapsto b^{i}(t, x)$ are continuous for each $t \in[0,1)$ and are bounded on bounded sets in $[0,1) \times \mathbb{R}^{d}$. In addition, suppose that, for every fixed ball $U \subset \mathbb{R}^{d}$, the functions $x \mapsto a^{i j}(t, x), t \in[0,1)$, are equicontinuous on $U$. Finally, assume that there exists a nonnegative compact function $\Psi \in C^{2}\left(\mathbb{R}^{d}\right)$ and a constant $C \geq 0$ such that

$$
L \Psi(t, x) \leq C+C \Psi(x) .
$$

Then, for every probability measure $\nu$, there exists a family $\mu=\left(\mu_{t}\right)_{t \in[0,1)}$ of probability measures on $\mathbb{R}^{d}$ satisfying (1.1) and (1.3) such that $t \mapsto \int_{\mathbb{R}^{d}} \zeta d \mu_{t}$ is continuous on $[0,1)$ for every $\zeta \in C_{0}^{\infty}\left(\mathbb{R}^{d}\right)$.

Moreover, if $\operatorname{det} A$ is separated from zero on compact subsets in $(0,1) \times \mathbb{R}^{d}$, then the continuity of $b$ in $x$ is not needed. 
It should be noted that the solutions constructed above possess nice global regularity properties. According to [7], if in the situation of Theorem 3.1 one has additionally that $A$ and $A^{-1}$ are uniformly bounded, $A$ is uniformly Lipschitzian in $x$, $|b|, \ln \left(|x|^{2}+1\right) \in L^{2}(\mu)$, and $\mu_{0}=\varrho_{0} d x$ with $\varrho_{0} \ln \varrho_{0} \in L^{1}\left(\mathbb{R}^{d}\right)$, then $\mu=\varrho(t, x) d x d t$ and

$$
\int_{0}^{\tau} \int_{\mathbb{R}^{d}} \frac{|\nabla \varrho(t, x)|^{2}}{\varrho(t, x)} d x d t<\infty
$$

for all $\tau<1$. The assumption that $|b|+\ln \left(|x|^{2}+1\right) \in L^{2}\left(\left.\mu\right|_{[0, \tau) \times \mathbb{R}^{d}}\right)$ is fulfilled if $\|A(t, x)\| \leq \alpha, \quad|b(t, x)| \leq c_{0}+c_{0} \exp \left(c|x|^{2 k} / 2\right), \quad\langle b(t, x), x\rangle \leq \gamma-(2 \alpha c k+\varepsilon)|x|^{2 k}$ with some $\alpha, c_{0}, c, k, \gamma>0$. If the latter estimate is replaced by

$$
\langle b(t, x), x\rangle \leq \gamma-\gamma_{0}|x|^{2 k+\delta}
$$

with positive $\gamma_{0}$ and $\delta$ and $\varrho_{0}$ is bounded, then $\varrho$ is uniformly bounded on every set $[0, \tau) \times \mathbb{R}^{d}, \tau<1$. Moreover, certain pointwise bounds on $\varrho$ are obtained in [7].

Aknowledgement. This work has been supported in part by the RFBR project 04-01-00748, the INTAS project 99-559, the DFG Grant 436 RUS 113/343/0(R), SFB 701 at the University of Bielefeld, the research programme "Analisi e controllo di equazioni di evoluzione deterministiche e stocastiche" from the Italian "Ministero della Ricerca Scientifica e Tecnologica", the Russian-Japanese Grant 05-01-02941JF. Most of the work was done during visits of the first and third authors to the Scuola Normale Superiore di Pisa and visits of the first and second authors to the University of Bielefeld.

\section{REFERENCES}

[1] V.I. Bogachev, G. Da Prato, M. Röckner, Existence of solutions to weak parabolic equations for measures, Proc. London Math. Soc. (3) 88 (2004), no. 3, 753-774.

[2] V.I. Bogachev, N.V. Krylov, M. Röckner, Differentiability of invariant measures and transition probabilities of singular diffusions, Dokl. Russian Acad. Sci. 376 (2001), n 2, 151-154.

[3] V.I. Bogachev, N.V. Krylov, M. RöCKner, On regularity of transition probabilities and invariant measures of singular diffusions under minimal conditions, Comm. Partial Diff. Equations 26 (2001), no. 11-12, 2037-2080.

[4] V.I. Bogachev, M. Röckner, Elliptic equations for infinite dimensional probability distributions and Lyapunov functions, C. R. Acad. Sci. Paris, sér. 1, 329 (1999), 705-710.

[5] V.I. Bogachev, M. RöckneR, A generalization of Hasminskii's theorem on existence of invariant measures for locally integrable drifts, Theory Probab. Appl. 45 (2000), no. 3, 417-436.

[6] V.I. Bogachev, M. Röckner, Elliptic equations for measures on infinite-dimensional spaces and applications, Probab. Theory Related Fields 120 (2001), no. 4, 445-496.

[7] V.I. Bogachev, M. Röckner, S.V. Shaposhnikov, Global regularity and bounds for solutions of parabolic equations for probability measures, Preprint SFB 701 N 05-011 (2005), Bielefeld University; Theory Probab. Appl. 50 (2005), no. 4, 652-674.

[8] V.I. Bogachev, M. Röckner, W. Stannat, Uniqueness of solutions of elliptic equations and uniqueness of invariant measures of diffusions, Sbornik Math. 193 (2002), no. 7, 945-976.

[9] V.I. Bogachev, M. Röckner, F.-Y. WANG, Elliptic equations for invariant measures on finite and infinite dimensional manifolds, J. Math. Pures Appl. 80 (2001), 177-221.

[10] J. Dohmann Feller-type properties and path regularities of Markov processes. Forum Math. 17 (2005), no. 3, 343-360.

[11] N.V. KRYLOV, An analytic approach to SPDEs, Stochastic partial differential equations: six perspectives, R. Carmona, B. Rozovskii, Eds.; Amer. Math. Soc.: Rhode Island, Providence, 1999, pp. $185-241$.

[12] O.A. Ladyz'enskaya, V.A. Solonnikov, N.N. Ural'tseva, Linear and quasilinear equations of parabolic type, Amer. Math. Soc., Rhode Island, 1968.

[13] G.M. Lieberman, Second order parabolic differential equations, World Scientific, Singapore, 1996. 
[14] W. Stannat, (Nonsymmetric) Dirichlet operators on $L^{1}$ : existence, uniqueness and associated Markov processes, Annali Scuola Normale Super. di Pisa Cl. Sci. (4) 28 (1999), no. 1, 99-140.

[15] W. Stannat, Time-dependent diffusion operators on $L^{1}$, Preprint SFB 343, Univ. Bielefeld, N 00-080 (2000), 41 pp.; J. Evol. Equat. 4 (2004), no. 4, 463-495.

Department of Mechanics and Mathematics, Moscow State University, 119899 Moscow, Russia

E-mail address: bogachev@vbogach.mccme.ru

Scuola Normale Superiore di Pisa, Piazza dei Cavalieri 7, I-56126 Pisa, Italy

E-mail address: daprato@sns.it

Fakultät Für Mathematik, Universität Bielefeld, D-33501 Bielefeld, Germany

E-mail address: roeckner@mathematik.uni-bielefeld.de 